\title{
AN EARTH-FLATTENING TRANSFORMATION FOR WAVES FROM A POINT SOURCE*
}

\author{
By DAVID P. HILL
}

\begin{abstract}
An earth-flattening transformation is developed for wave-propagation problems that can be formulated in terms of uncoupled scalar Helmholtz equations. Through the transformation, wave problems in isotropic, spherically symmetric media with a specified radial heterogeneity can be expressed in terms of a flat geometry with a suitably vertical heterogeneity. The transformation is exact for homogeneous (no source) problems and is useful for normal mode studies. When a point source of waves is present, the earth-flattening transformation together with the Watson transform converts the reflected wave field from a sum over discrete, spherical eigenfunctions to an integral over continuous wave numbers in a flat geometry. The far-field form of this integral shares many properties with the Weyl integral and is useful for body-wave studies in a spherical earth.
\end{abstract}

\section{INTRODUCTION}

Earth-flattening transformations provide a means for including the Earth's curvature in plane layer formulations of wave-propagation problems. Through such transformations, the existing store of analytical and numerical methods for treating flat problems become available for treating problems with spherical geometry. With emphasis on the resolution of progressively more subtle details of the Earth's structure using both phase and amplitude information on seismograms, it becomes important to include the effects of curvature for even relatively short propagation paths and wave lengths.

Earth-flattening approximations based on a linear modification of the index of refraction have been used for some time in making corrections for the Earth's curvature in radio-wave propagation problems (Schelling et al., 1933; Budden, 1960). Alterman et al. (1961) introduced an analogous approximation into the seismological literature for short-period Love waves. Kovach and Anderson (1962) and Anderson and Toksöz (1963) developed a more general earth-flattening transformation for Love waves that involves transforming radially symmetric, isotropic shells into flat, vertically heterogeneous, anisotropic layers. More recently, Satô (1968) and Biswas and Knopoff (1970) introduced transformations that exactly convert toroidal, or $S H$-wave motion in a spherically symmetric, radially heterogeneous earth to $S H$ motion in plane, vertically heterogeneous layers. The exact earth-flattening transformations developed by these authors for homogeneous (no sources) equations of $S H$ motion are most useful for normal mode or surface-wave problems. Analogous exact transformations for homogeneous spheroidal, or $P-S V$ wave motion have not been reported; one might expect that such a transformation cannot be made exactly because the velocity gradients that serve to map spherical to plane geometries would introduce spurious $P-S V$ coupling.

Muller (1971) has developed an approximate earth-flattening transformation for body waves using arguments based on geometric ray theory. In this paper, we develop an earth-flattening transformation suitable for body waves from a wave-theoretical approach by incorporating an exact flattening transformation into the inhomogeneous

*Publication authorized by the Director, U.S. Geological Survey. 
equations of motion for waves generated by a steady point source in a radially heterogeneous earth. The transformation is developed for wave problems that can be formulated in terms of uncoupled scalar Helmholtz equations in spherically symmetric, radially heterogeneous media. Because acoustic, electromagnetic, and elastic wave propagation problems can be expressed in terms of uncoupled Helmholtz equations under a wide range of conditions, this earth-flattening operator applies to a variety of geophysical applications. Analogous earth-flattening transformations are readily developed for any wave-propagation problem in which the equations of motion are separable in a spherical coordinate system and in which the radial eigenfunctions are associated with a Sturm-Liouville operator.

Our approach will be first to summarize briefly the potential representations known to reduce the acoustic and elastic equations of motion in radially heterogeneous media to canonical wave equations (or Helmholtz equations in the steady state). Because a primary motivation for this paper is to obtain an earth-flattening transformation for seismic body waves, we give particular attention to the elastic displacement potentials recently introduced by Richards (1971) that result in high-frequency decoupling of $P-S V$ motion in both radially and vertically heterogeneous media.

Next, we develop an exact earth-flattening transformation for the homogeneous Helmholtz equation for waves in a spherical medium in which the wave velocity and density may vary smoothly with radius (this transformation is parallel to those obtained by Satô (1968) and Biswas and Knopoff (1970) for the $S H$-wave equation). In addition, we will indicate how the continuity conditions across an abrupt change in velocity and density behave under the transformation using $S H$ motion as an example.

Finally, taking acoustic motion as a simple example and introducing the exact earthflattening transformation together with the Watson transform, we convert the response of a radially heterogeneous body to a steady point source from an infinite sum over discrete order numbers to an integral over continuous wave numbers in a vertically heterogeneous half-space. Here we will show that for conditions generally satisfied by body waves in the crust and upper mantle, this integral reduces to the form of a Weyl (or Sommerfield) integral in which the effects of curvature are included in a generalized reflection coefficient.

\section{Equations of Motion and Potential Representations}

We will restrict our considerations to plane layered and spherically layered heterogeneous, isotropic media. Specifically, we will consider acoustic and elastic isotropic media in which the scalar material parameters may vary as a function of either depth or radius beneath the reference surface $z=0$ or $r=a$ (across which the material parameters change discontinuously). Furthermore, we will consider a single, isotropic steady point source of angular frequency, $\omega$, so that the resulting field depends on only two coordinates ( $z$ and $\rho$ in a cylindrical system and $r$ and $\theta$ in a spherical system, see Figures 1 and 2).

In inviscid fluid media, the acoustic approximation to the equations of motion can be written as

$$
\nabla^{2} P+k^{2} P-\rho^{-1} \nabla P \cdot \nabla \rho=0
$$

where $P$ is the deviation from the static ambient pressure, $\rho$ is the density and $k=\omega / c$, $c$ being the acoustic velocity. Following Brekhovskikh (1960), we introduce the pressure potential

$$
\psi=P \rho^{-\frac{1}{2}}
$$




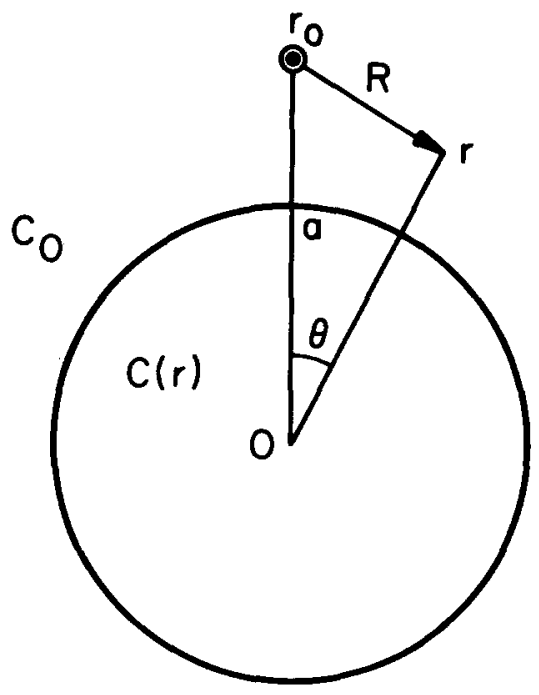

FIG. 1. Coordinate system for a spherically symmetric medium with a boundary at $r=a$ between a homogeneous region $\left(C_{0}\right)$ and a radially heterogeneous region $(C(r))$. The point source and receiver are located at $r_{0}$ and $r$, respectively.

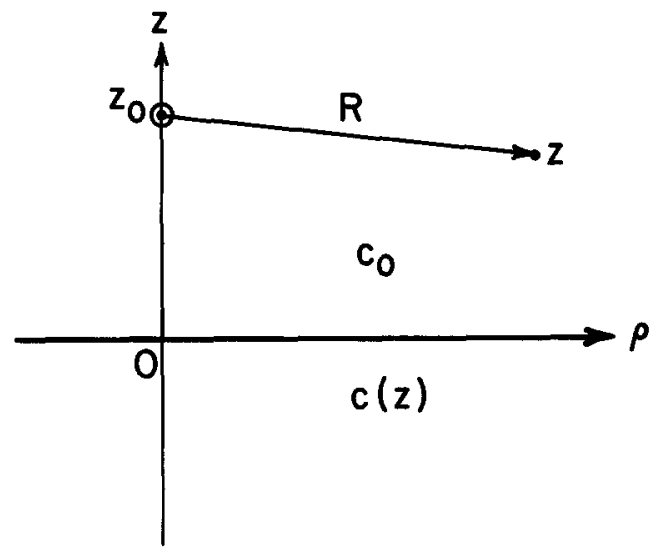

FIG. 2. Coordinate system for a cylindrically symmetric medium with a boundary at $z=0$ between a homogeneous region $\left(C_{0}\right)$ and a vertically heterogeneous region $(C(z))$. The point source and receiver are located at $z_{0}$ and $z$, respectively.

which reduces (1) to the form of a Helmholtz equation

$$
\nabla^{2} \psi+k^{2} \psi=0
$$

In obtaining (3) the assumption is made that

$$
\left|k^{2}\right| \geqslant\left|\frac{1}{2 \rho} \nabla^{2} \rho-\frac{3}{4}\left(\frac{\nabla \rho}{\rho}\right)^{2}\right|
$$

i.e. the density gradients are small with respect to the wave number, $k$. In this representation a steady point source of pressure, $\mathscr{F}$, is given by the potential

$$
F=\mathscr{F} \rho^{-\frac{1}{2}}
$$

where

$$
\mathscr{F}=\frac{\delta\left(r-r_{0}\right) \delta\left(\theta-0_{+}\right) e^{-i \omega t}}{2 \pi r^{2} \sin \theta}
$$

in a spherical coordinate system. 
Richards (1970) has developed potential representations for the vector-wave equation in radially heterogeneous and vertically heterogeneous (in spherical and Cartesian systems, respectively) isotropic, elastic media. From his formulation, he is able to show that coupled $P$ and $S V$ solutions exist for all possible displacement solutions, $S H$ solutions are decoupled from the $P-S V$ solutions, and, at sufficiently high frequencies, $P-S V$ solutions tend to decouple into forms that can be identified with the standard irrotational $P$-wave and solenoidal $S V$-wave solutions in homogeneous media. The last two results are particularly useful here because they permit formulation of the radially or vertically inhomogeneous elastic wave propagation problems in terms of three uncoupled Helmholtz equations for frequencies commonly encountered in crustal and upper-mantle body-wave studies.

In a spherically symmetric system, Richard's displacement potentials for $P, S V$, and $S H$ motion ( $P, S$, and $T$, respectively) have the following relation to the displacement vector, $\mathbf{U}(r)$, at $\mathbf{r}=(r, \theta, \phi)$

$$
\begin{aligned}
\mathbf{U}(\mathbf{r})= & {[f(r)]^{-1}\left\{\operatorname{grad}\left(\frac{f(r)}{[\rho(r)]^{\frac{1}{2}}} P(\mathbf{r})\right)\right.} \\
& \left.+\operatorname{curl} \operatorname{curl}\left(\frac{r f(r)}{[\rho(r)]^{\frac{1}{2}}} S() \mathbf{r}, 0,0\right)\right\} \\
& +\operatorname{curl}\left(\frac{r}{[\mu(r)]^{\frac{1}{2}}} T(\mathbf{r}), 0,0\right)
\end{aligned}
$$

where $\rho(r)$ and $\mu(r)$, the density and shear modulus, are functions of radius and the scale factor, $f(r)$, is any sufficiently smooth, bounded function of radius. The associated source potentials are related to the applied force per unit mass, $\mathbf{F}$, by

$$
\begin{aligned}
\mathbf{F}= & f^{-1}\left\{\operatorname{grad}\left(\frac{f}{\rho^{\frac{1}{2}}} D\right)+\operatorname{curl} \operatorname{curl}\left(\frac{r f}{\rho^{\frac{1}{2}}} F, 0,0\right)\right\} \\
& +\operatorname{curl}\left(\frac{r}{\mu^{\frac{1}{2}}} E, 0,0\right)
\end{aligned}
$$

The spheroidal $(P-S V)$ equations of motion can be written in terms of $P(\mathbf{r})$ and $S(\mathbf{r})$ as a fourth and fifth-order pair of coupled equations. Richards (1971) shows that if the scaling factor, $f(r)$, is chosen such that

$$
\left(\frac{\lambda+\mu}{\lambda+2 \mu}\right) \frac{f^{\prime}}{f}=\frac{\rho^{\prime}}{\rho}+\frac{2 \mu^{\prime}}{\lambda+2 \mu},
$$

where $\lambda$ and $\mu$ are Lamé parameters and the prime indicates differentiation with respect to radius, and if the source potential is of the form

$$
D=\frac{\delta\left(r-r_{0}\right) \delta\left(\theta-0_{+}\right) e^{i \omega t}}{2 \pi r^{2} \sin \theta}, \quad F=0, \quad E=0
$$

then the following relations for $P$ and $S$ are both sufficient and necessary for highfrequency decoupling of the $P$ and $S$ potentials in the spheroidal equations of motion

and

$$
\frac{\rho D}{\lambda+2 \mu}+\nabla^{2} P+\frac{\rho \omega^{2}}{\lambda+2 \mu} P=0(1) \cdot P
$$

$$
\nabla^{2} S+\frac{\rho \omega^{2}}{\mu} S=0
$$


For sufficiently high frequencies, (6) can be written

$$
\nabla^{2} P+\frac{\rho \omega^{2}}{\lambda+2 \mu} P \simeq-\frac{\rho D}{\lambda+2 \mu}
$$

where the $S$-coupled potential is two orders down in frequency with respect to $P$. In other words, for an appropriate source of compressional energy and for sufficiently high frequencies, the $P$ and $S$ displacement potentials approximately satisfy the separate Helmholtz equations (7) and (8).

The toroidal, or $S H$, equation of motion is simply

$$
\nabla^{2} T+\frac{\rho \omega^{2}}{\mu} T+\varepsilon_{T}(r) T=-\frac{\rho}{\mu} E
$$

for a steady source of the form

$$
E=\frac{\delta\left(r-r_{0}\right) \delta\left(\theta-0_{+}\right)}{2 \pi r^{2} \sin \theta} e^{i \omega t}, \quad D=0, \quad F=0
$$

where

$$
\varepsilon_{T}(r)=\frac{1}{4}\left(\frac{\mu^{\prime}}{\mu}\right)^{2}+\frac{1}{2} \frac{\mu^{\prime \prime}}{\mu}-\frac{2 \mu^{\prime}}{r \mu}
$$

(Richards, 1971). If we assume that shear modulus gradients are small with respect to the $S H$-wave number so that

$$
\left|\varepsilon_{T}\right| \ll\left|\frac{\rho \omega^{2}}{\mu}\right|
$$

then the torsional potential satisfies the Helmholtz equation

$$
\nabla^{2} T+\frac{\rho \omega^{2}}{\mu} T=-\frac{\rho}{\mu} E .
$$

Note that the assumption of small shear modulus gradients is analogous to the assumption of small density gradients made in obtaining the Helmholtz representation for acoustic motion(3).

The displacement potential representations for a vertically heterogeneous Cartesian system are similar to those for a spherical system. In particular, the displacement and source vectors are represented at the point $\mathbf{r}=(x, y, z)$ by

$$
\begin{aligned}
\mathbf{U}(\mathbf{r})= & {[f(z)]^{-1}\left\{\operatorname{grad}\left(\frac{f(z)}{[\rho(z)]^{\frac{1}{2}}} \mathbf{P}(\mathbf{r})\right)+\operatorname{curl} \operatorname{curl}\left(0,0, \frac{f(z)}{[\rho(z)]^{\frac{1}{2}}} S(\mathbf{r})\right)\right\} } \\
& +\operatorname{curl}\left(0,0,[\mu(z)]^{-\frac{1}{2}} T(\mathbf{r})\right)
\end{aligned}
$$

and

$$
\begin{aligned}
\mathscr{F}= & f^{-1}\left\{\operatorname{grad}\left(\frac{f}{\rho^{\frac{1}{2}}} D\right)+\operatorname{curl} \operatorname{curl}\left(0,0, \frac{f}{\rho^{\frac{1}{2}}} F\right)\right\} \\
& +\operatorname{curl}\left(0,0, \mu^{-\frac{1}{2}} E\right) .
\end{aligned}
$$

These potentials decouple at high frequencies in the same manner as those in the spherical system. Equations (7), (8), and (9) apply to both systems.

Richards (1971) points out that the commonly chosen potential representation for $P-S V$ motion

$$
\mathbf{U}=\operatorname{grad} \Phi+\operatorname{curl} \operatorname{curl}(0,0, X)
$$


where $\Phi$ and $X$ are assumed to satisfy

$$
\nabla^{2} \Phi+\frac{\rho(z) \omega^{2}}{\lambda(z)+{ }^{2} \mu(z)} \Phi=0, \quad \nabla^{2} X+\frac{\rho(z) \omega^{2}}{\mu(z)} X=0
$$

does not lead to decoupled equations in $\Phi$ and $X$ at high frequencies except in the special case of constant density. If we are to allow for the effects of a variable density, the potential representations (4) or (10) must be used.

\section{The TRANSFORMation}

Consider a spherically symmetric medium that is homogeneous for $r>a$ and radially heterogeneous for $r<a$ and in which the scalar field, $\psi$, satisfies the Helmholtz equation. If a point source is located in the homogeneous medium at $r=r_{0}\left(r_{0}>a\right)$ and $\theta=0_{+}$, the field in the homogeneous medium $\psi_{>}$, will be given by

$$
\nabla^{2} \psi_{>}+k_{0}^{2} \psi_{>}=-\frac{\delta\left(r-r_{0}\right) \delta\left(\theta-0_{+}\right)}{2 \pi r^{2} \sin \theta} .
$$

Inside the boundary $r=a$, the field $\psi_{<}$will be given by

$$
\nabla^{2} \psi_{<}+k^{2}(r) \psi_{<}=0
$$

where $k_{0}=$ const and $k=k(r)$ (see Figure 1). The general solution to (12a) in $r>a$ will be

$$
\psi_{>}=\psi_{0}+\psi_{p}
$$

where $\psi_{0}$ is the solution to the homogeneous equation, which includes the effects of both a boundary at $r=a$ and the underlying heterogeneous medium in a reflection coefficient, and $\psi_{p}$ is a particular solution to the inhomogeneous equation.

An appropriate particular solution to (12a) is the infinite space Green's function, or

$$
\psi_{p}=\frac{e^{i k R}}{R}
$$

where $R$ is the straight line distance between the source at $r=\left(r_{0}, 0_{+}\right)$and any point $\mathbf{r}=(r, \theta)$.

We apply a Legendre transform to $(12 a, b)$, where the Legendre transform pair is defined by

$$
\begin{aligned}
\hat{\psi}_{l}(r) & =\int_{0}^{\pi} \psi(r, \theta) P_{l}(\cos \theta) \sin \theta d \theta \\
\psi(r, \theta) & =\sum_{l=0}^{\infty}\left(l+\frac{1}{2}\right) \hat{\psi}_{l}(r) P_{l}(\cos \theta),
\end{aligned}
$$

to obtain

$$
\left[\frac{d}{d r^{2}}+\frac{2}{r} \frac{d}{d r}+\left(k^{2}-\frac{l(l+1)}{r^{2}}\right)\right]\left[\begin{array}{l}
\hat{\psi}_{>1} \\
\hat{\psi}_{<1}
\end{array}\right]=\left[\begin{array}{c}
-\frac{\delta\left(r+r_{0}\right)}{2 \pi r^{2}} \\
0
\end{array}\right]
$$

\section{Homogeneous CASE}

Our aim is to rearrange the homogeneous form of (16) so that it resembles the analogous differential equation in $z$ for a cylindrical system, $\mathbf{r}=(\rho, \Phi, z)$, or

$$
\left[\frac{d^{2}}{d z^{2}}+\left(k_{f}^{2}-\kappa^{2}\right)\right] \Phi=0 .
$$


To accomplish this, we transform the independent variable in (16) according to

$$
-z=a \ln (a / r)
$$

and the dependent variable according to

$$
\hat{\psi}_{l}=\Phi_{l}\left(\frac{a}{r}\right)^{\frac{1}{2}}=\Phi_{l} e^{-z / 2 a} .
$$

Putting these two expressions into the homogeneous form of (16) gives

$$
\left\{\frac{d^{2}}{d z^{2}}+\left[k^{2}\left(\frac{r}{a}\right)^{2}-\frac{l(l+1)+\frac{1}{4}}{a^{2}}\right]\right\} \Phi_{l}=0
$$

which is the desired result.

A direct comparison between (20) and (17) yields the following exact earth-flattening transformation for the homogeneous Helmholtz equation

$$
\begin{aligned}
\kappa_{f} & =k\left(\begin{array}{l}
r \\
a
\end{array}\right) \\
\kappa^{2} & =\frac{l(l+1)+\frac{1}{4}}{a^{2}}
\end{aligned}
$$

with

$$
\begin{gathered}
z=a \ln (r / a), \quad r=a e^{z / a} \\
\Phi_{l}=\left(\begin{array}{c}
r \\
\frac{a}{a}
\end{array}\right)^{\frac{1}{2}} \hat{\psi}_{l}
\end{gathered}
$$

where $k_{f}$ and $k$ are the flat and spherical wave numbers, respectively. The expression in the brackets multiplying $\Phi_{l}$ in (20) is the radial wave number component in the spherical case. Thus, we identify $\kappa$ in (21) as the angular wave number component, and from this we obtain the well-known relation

$$
\kappa a=l+\frac{1}{2} .
$$

The propagation velocity in the medium is given by $c=\omega / k$, giving

$$
c_{f}=c_{s} e^{-z / a}
$$

where $c_{f}$ and $c_{s}$ refer to the velocities in the flat and spherical systems, respectively. If we are interested in the field in the vicinity of $r=a$, the above transformation can be approximated by

$$
\begin{aligned}
c_{f} & \simeq c_{s}(1-z / a), \\
r & \simeq a(1+z / a),
\end{aligned}
$$

This represents the earth-flattening approximation used by earlier workers (Budden, 1960).

Our development is similar to that of Biswas and Knopoff (1970) or Satô (1968) for their exact transformation of the homogeneous equation for $S H$ motion, although the particular potential representation and initial differential equations used result in minor differences in the transformation. The transformations for depth (21) and velocity (22) are the same as those obtained by Muller (1971) from geometrical ray theory. 


\section{Relation Between Spherical and Plane Boundary Conditions}

In making the above transformation, we have implicitly assumed that the properties of the medium vary smoothly with radius. If we allow a discontinuous jump in properties at some level, say $r=a$, it is necessary to consider how the continuity (boundary) conditions for this jump transform. To illustrate this, we will compare the spherical and flat boundary conditions for $S H$ waves under the above transformation.

Continuity of both displacement and stress are required across a welded boundary. For $S H$ (or torsional) motion across a spherical boundary, these conditions are

for displacement and

$$
\left[U_{\phi}\right]_{a+}=\left[U_{\phi}\right]_{a-}
$$

$$
\left[\mu\left(\frac{\partial U_{\phi}}{\partial r}-\frac{U_{\phi}}{r}\right)\right]_{a+}=\left[\mu\left(\frac{\partial U_{\phi}}{\partial r}-\frac{U_{\phi}}{r}\right)\right]_{a-}
$$

for stress. The displacement potential representation for $S H$ motion in a spherical system is given by the last term in (4) or

$$
|\mathbf{U}|=U_{\phi}=-\frac{1}{r} \frac{\partial}{\partial \theta}\left(\frac{r}{\mu^{\frac{1}{2}}} T\right) .
$$

The potential, $T$, satisfies the Helmholtz equation (7), and its eigenfunctions in a spherical system are of the form

$$
T_{l}(r, \theta)=\left(l+\frac{1}{2}\right) \hat{T}_{l}(r) P_{l}(\cos \theta)
$$

where $\hat{T}_{l}(r)$ is the homogeneous solution to (16). By (21), the mapping of $\hat{T}(r)$ into the flat potential, $\hat{T}_{f}(z)$, is given by

$$
\begin{aligned}
\hat{T}(r) & =\left(\frac{a}{r}\right)^{\frac{1}{2}} \hat{T}_{f}(z) \\
\left(\frac{a}{r}\right)^{\frac{1}{2}} & =e^{-z / 2 a}
\end{aligned}
$$

From this, the exact boundary conditions in a spherical system expressed in terms of the flat potential, $\hat{T}_{f}(z)$ are

$$
\begin{aligned}
{\left[\mu^{-\frac{1}{2}} \hat{T}_{f}\right]_{0+} } & =\left[\mu^{-\frac{1}{2}} \hat{T}_{f}\right]_{0-} \\
{\left[\frac{\mu^{\prime}}{\mu^{\frac{1}{2}}}-\mu^{\frac{1}{2}}\left(\hat{T}_{f^{\prime}}-\frac{3}{2} \frac{\hat{T}_{f}}{a}\right)\right]_{0+} } & =\left[\frac{\mu^{\prime}}{\mu^{\frac{1}{2}}}-\mu^{\frac{1}{2}}\left(\hat{T}_{f}^{\prime}-\frac{3}{2} \frac{\hat{T}_{f}}{a}\right)\right]_{0-}
\end{aligned}
$$

where the primes indicate differentiation with respect to $z$.

On the other hand, the analogous continuity conditions for a flat boundary are

$$
\left[U_{\phi}\right]_{0+}=\left[U_{\phi}\right]_{0-}
$$

for displacement ( $\phi$ represents the angular coordinate in a cylindrical system or the coordinate in a Cartesian system), and

$$
\left\{\mu \frac{\partial}{\partial z} U_{\phi}\right\}_{0+.}=\left\{\mu \frac{\partial}{\partial z} U_{\phi}\right\}_{0-}
$$

for stress ( $\rho$ represents the radial coordinate in a cylindrical system). In this case, $T_{p}$ is the displacement potential for a plane system given by the last term in (10), or

$$
|\mathbf{U}|=U_{\phi}=-\frac{\partial}{\partial \rho} \frac{T_{p}}{\mu^{\frac{1}{2}}}
$$


Again, the potential, $T_{p}$, satisfies the Helmholtz equation, and its eigenfunctions in a cylindrical system are

$$
T_{p}=\hat{T}_{p}(z) J_{0}(\kappa \rho)
$$

The above continuity conditions become

$$
\begin{gathered}
{\left[\mu^{-\frac{1}{2}} \hat{T}_{p}\right]_{0+}=\left[\mu^{-\frac{1}{2}} \hat{T}_{p}\right]_{0-}} \\
{\left[\frac{\mu^{\prime}}{\mu^{\frac{1}{2}}}-\mu^{\frac{1}{2}} \hat{T}_{p}^{\prime}\right]_{0+}=\left[\frac{\mu^{\prime}}{\mu^{\frac{1}{2}}}-\mu^{\frac{1}{2}} \hat{T}_{p}^{\prime}\right]_{0-}}
\end{gathered}
$$

where, again, the primes indicate differentiation with respect to $z$. Comparing (25) with (24), we see that the boundary conditions for the spherical boundary expressed in terms of the earth-flattening transformation are similar to the boundary conditions for the plane boundary except for the term of order $\left(\hat{T}_{f} / a\right)$ subtracted from $\hat{T}_{f}^{\prime}$ in the stress condition in the spherical case. Because the two potentials $\hat{T}_{p}$ and $\hat{T}_{f}$ enter the boundary conditions in the same way, it is possible to use the plane-boundary formulation and potential representation to obtain solutions for the spherical boundary problem, although for an exact spherical solution, conditions (24) must be used rather than the natural conditions for a plane boundary, (25). However, we note that

$$
\hat{T}_{f}^{\prime}=0\left([1 / \eta] \hat{T}_{f}\right)
$$

where $\eta$ is a vertical wave number. The additional term in the spherical stress-boundary condition (24) is thus of the order $(\eta a)^{-1}$ with respect to $\hat{T}_{f}$. In most crustal and uppermantle body-wave problems $\left|(\eta a)^{-1}\right|$ will be small (provided we avoid grazing angles of incidence), and the natural plane boundary conditions (25) can be used with little loss in accuracy. Similar remarks hold for the transformation of boundary conditions for the decoupled P-SV system in terms of Richards' potentials and for acoustic motion (Hill, 1971).

\section{The Point Source}

We now turn to the development of an approximate earth-flattening transformation when a point source of spherical waves is included. To keep the algebra to a minimum, we will use the acoustic case as an example; the elastic and $S H$ and $P-S V$ cases follow in a parallel manner.

The model is shown in Figure 1 and the Legendre-transformed field equations are given by (16). Here we take $\psi$ to be the pressure potential defined by (2). Using equations (10.1.45) and (10.1.46) in Abramowitz and Stegun (1964), the particular solution (14) can be represented as

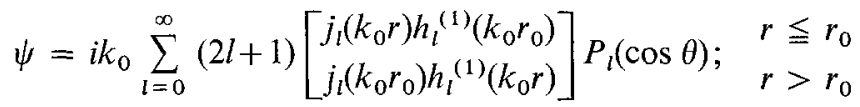

where $j_{l}$ and $h_{l}{ }^{(1)}$ are spherical Bessel and Hankel functions and $P_{l}$ is a Legendre polynomial. By comparing the form of the sum in the inverse Legendre transform (15b) with this series representation of the particular solution above, we see that

$$
\hat{\psi}_{l}=2 i k_{0} \begin{cases}j_{l}\left(k_{0} r\right) h_{l}^{(1)}\left(k_{0} r_{0}\right) & r \leqq r_{0} \\ j_{l}\left(k_{0} r_{0}\right) h_{l}^{(1)}\left(k_{0} r\right) & r>r_{0} .\end{cases}
$$

Accordingly, the general form of the solution can be written as

$$
\begin{array}{ll}
\hat{\psi}_{>l}(r)=2 i k_{0} h_{l}^{(1)}\left(k_{0} r_{0}\right) j_{l}\left(k_{0} r\right)+A h_{l}^{(1)}\left(k_{0} r\right) ; & a<r \leqq r_{0} \\
\hat{\psi}_{<l}(r)=B\left(\frac{a}{r}\right)^{\frac{1}{2}} \Phi_{l}(z) ; & r<a .
\end{array}
$$


The first term in the solution for $r>a$ is the source field (26), and the second term is the field scattered by the sphere. In the second term, $A$ is a constant determined by the boundary conditions and $h_{l}^{(1)}\left(k_{0} r\right)$ is a solution to (16) that represents outward traveling waves when $k_{0} r \gg 1$. We represent the solution to the field inside the sphere, which may be radially heterogeneous, in terms of the exact earth-flattening transformation (21).

The boundary conditions at the surface of the sphere, $r=a$, require continuity of pressure and the normal component of particle velocity for an acoustic field, or

$$
\begin{aligned}
{\left[\rho_{0}^{\frac{1}{2}} \hat{\psi}_{>l}\right]_{a+} } & =\left[\rho_{1}^{\frac{1}{2}} \hat{\psi}_{<l}\right]_{a-} \\
{\left[\rho_{0}{ }^{-1} \frac{d}{d r}\left(\rho_{0}^{\frac{1}{2}} \hat{\psi}_{>l}\right)\right]_{a+} } & =\left[\rho_{1}^{-1} \frac{d}{d r}\left(\rho_{1}^{\frac{1}{2}} \hat{\psi}_{<l}\right)\right]_{a-} .
\end{aligned}
$$

Putting (27) into these boundary conditions gives

$$
\left[\begin{array}{cc}
\rho_{0}{ }^{\frac{1}{2}} h_{l}^{(1)}\left(k_{0} r\right) & -\rho_{1}^{\frac{1}{2}}\left(\frac{a}{r}\right)^{\frac{1}{2}} \Phi_{l} \\
\rho_{0}{ }^{-\frac{1}{2}} \frac{d}{d r} h_{l}^{(1)}\left(k_{0} r\right) & -\rho_{1}^{-1} \frac{d}{d r}\left[\rho_{1}^{\frac{1}{2}}\left(\frac{a}{r}\right)^{\frac{1}{2}} \Phi_{l}\right]
\end{array}\right]\left[\begin{array}{c}
A \\
B
\end{array}\right]=-S\left[\begin{array}{c}
\rho_{0}^{\frac{1}{2}} j_{l}\left(k_{0} r\right) \\
\rho_{0}^{-\frac{1}{2}} \frac{d}{d r} j_{l}\left(k_{0} r\right)
\end{array}\right]
$$

where $S=2 i k_{0} h_{l}^{(1)}\left(k_{0} r_{0}\right)$. Solving this system for $A$ we obtain

$$
A=i k_{0} h_{l}^{(1)}\left(k_{0} r_{0}\right) \frac{j_{l}\left(k_{0} a\right)}{h_{l}^{(1)}\left(k_{0} a\right)} V,
$$

where $V$ can be regarded as a generalized spherical reflection coefficient given by

$$
V=\left[\frac{\rho_{0}^{\frac{1}{2}} \rho_{1}^{-1} \frac{d}{d r}\left[\left(\rho_{1} \frac{a}{r}\right)^{\frac{1}{2}} \Phi_{l}\right]-\left(\frac{\rho_{1}}{\rho_{0}}\right)^{\frac{1}{2}} \Phi_{l} k_{0} \frac{j_{l}\left(k_{0} r\right)}{j_{l}\left(k_{0} r\right)}}{\rho_{0}^{\frac{1}{2}} \rho_{1}^{-1} \frac{d}{d r}\left[\left(\rho_{1} \frac{a}{r}\right)^{\frac{1}{2}} \Phi_{l}\right]+\left(\frac{\rho_{1}}{\rho_{0}}\right)^{\frac{1}{2}} \Phi_{l} k_{0} \frac{h_{l}^{(1)}\left(k_{0} r\right)}{h_{l}^{(1)}\left(k_{0} r\right)}}\right]_{r=a}
$$

The expression for the spectral amplitude and phase of the external field can now be obtained by applying the inverse Legendre transform (15b) to $\hat{\psi}_{>l}$ in (27) with $A$ given by (29). It is necessary to sum the series

$$
\psi_{>}=\frac{1}{2} \sum_{l=0}^{\infty}(2 l+1) \hat{\psi}_{>l} P_{l}(\cos \theta) .
$$

This series is known to converge slowly for large $l$, which is the range of interest for body-wave studies. The standard way around this problem is to convert the sum into an integral using the Watson transform (see, for example, Bremner, 1949; Scholte, 1956; Chapman, 1969; or Gilbert and Helmberger, 1971). Accordingly, we obtain

$$
\psi_{>}=\frac{1}{2 i} \int_{c} \frac{v d v}{\cos (v \pi)} \hat{\psi}_{>}(v) P_{v-\frac{1}{2}}[\cos (\pi-\theta)]
$$

where $v=\kappa a=l+\frac{1}{2}, \hat{\psi}_{>}(v)=\hat{\psi}_{>l}$ and the contour, $c$, is shown in Figure 3. The integrand in (30) is odd since $\hat{\psi}_{>}(v)=\hat{\psi}_{>}(-v)$ and $P_{v-\frac{1}{2}}(X)=P_{-v-\frac{1}{2}}(X)$ so that the path, $C$, can be transformed into $C_{1}$ or $C_{2}$ as shown in Figure 3.

Our goal here is to recast the integral representation for spherical waves scattered by a radially heterogeneous sphere into a form that resembles the Weyl integral representation for spherical waves reflected by a vertically heterogeneous half-space shown in Figure 2, or

$$
\psi_{r}=\frac{i}{2} \int_{-\infty}^{\infty} H_{0}^{(1)}(\kappa \rho) V_{p}(\kappa) e^{i \eta\left(z+z_{0}\right)} \frac{\kappa}{\eta} d \kappa
$$


where

$V_{p}$ is the plane wave reflection coefficient for the half-space $z \leqq 0$

$\kappa$ is the horizontal wave number

$\eta$ is the vertical wave number

(see Brekhovskikh, 1960 or Grant and West, 1965).

We begin by decomposing the integrand in (30) into terms that can more readily be given a physical interpretation. Using the identity

$$
j_{l}\left(k_{0} r\right)=\frac{1}{2}\left[h_{l}^{(1)}\left(k_{0} r\right)+h_{l}^{(2)}\left(k_{0} r\right)\right]
$$

the spherical Bessel function in the source term can be separated into spherical Hankel functions representing outgoing and ingoing traveling waves. Introducing this separation into $\hat{\psi}_{>}(v)$ as it appears in (30), we obtain

$$
\hat{\psi}_{>}(v)=i k_{0} h_{v-\frac{1}{2}}^{(1)}\left(k_{0} r\right)\left[h_{v-\frac{1}{2}}^{(1)}\left(k_{0} r\right)+h_{v-\frac{1}{2}}^{(2)}\left(k_{0} r\right)\right]+\left(A_{1}+A_{2}\right) h_{v-\frac{1}{2}}^{(1)}\left(k_{0} r\right)
$$

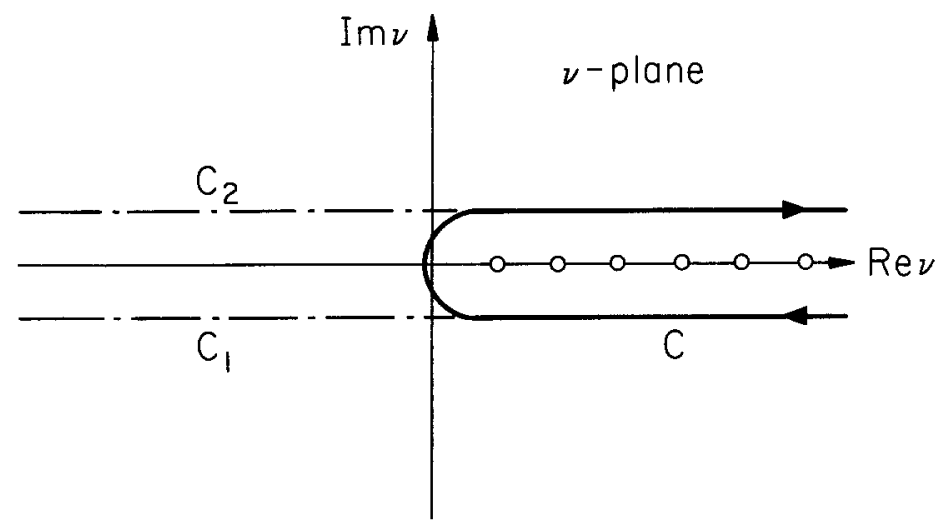

FIG. 3. Integration path for Watson transform in complex $v$ plane. The heavy line, $C$, is the original contour, and dashed lines, $C_{1}$ and $C_{2}$, are alternate positions for deformed contour.

where $A_{1}$ and $A_{2}$ are the parts of $A$ associated with the outgoing and ingoing waves from the source and are given by

$$
\begin{aligned}
& A_{1}=-i k_{0} h_{v-\frac{1}{2}}^{(1)}\left(k_{0} r\right) \\
& A_{2}=-i k_{0} h_{\nu-\frac{1}{2}}^{(1)}\left(k_{0} r\right) \frac{h_{v-\frac{1}{2}}^{(2)}\left(k_{0} a\right)}{h_{v-\frac{1}{2}}^{(1)}\left(k_{0} a\right)} V
\end{aligned}
$$

and where $V$ now has the form

$$
V=\frac{\rho_{0}^{\frac{1}{2}} \rho_{1}^{-1} \frac{d}{d r}\left[\left(\rho_{1} \frac{a}{r}\right)^{\frac{1}{2}} \Phi(v)\right]_{r=a}-\left(\frac{\rho_{1}}{\rho_{0}}\right)^{\frac{1}{2}} \Phi(v) k_{0} h_{2}}{-\rho_{0}^{\frac{1}{2}} \rho_{1}{ }^{-1} \frac{d}{d r}\left[\left(\rho_{1} \frac{a}{r}\right)^{\frac{1}{2}} \Phi(v)\right]_{r=a}+\left(\frac{\rho_{1}}{\rho_{0}}\right)^{\frac{1}{2}} \Phi(v) k_{0} h_{1}}
$$

with

$$
h_{1}=\left[h_{v-\frac{1}{2}}^{(1)^{\prime}}\left(k_{0} r\right) / h_{v-\frac{1}{2}}^{(1)}\left(k_{0} r\right)\right]_{r=a}
$$

and

$$
h_{2}=\left[h_{v-\frac{1}{2}}^{(2)}\left(k_{0}^{\prime} r\right) / h_{v-\frac{1}{2}}^{(2)}\left(k_{0} r\right)\right]_{r=a} .
$$


We see that the scattered field associated with the outgoing source field exactly cancels the outgoing source field (Phinney and Alexander, 1966), and we are left with

$$
\psi_{>}(v)=i k_{0} h_{\nu-\frac{1}{2}}^{(1)}\left(k_{0} r\right)\left[h_{v-\frac{1}{2}}^{(2)}\left(k_{0} r\right)+\frac{h_{v-\frac{1}{2}}^{(2)}\left(k_{0} a\right)}{h_{\nu-\frac{1}{2}}^{(1)}\left(k_{0} a\right)} h_{v-\frac{1}{2}}^{(1)}\left(k_{0} r\right) V\right]
$$

in the integral ( 30$)$.

Following Chapman (1969), we now introduce the representation for Legendre functions given by

$$
P_{\nu-\frac{1}{2}}[\cos (\pi-\theta)]=-i e^{i \pi v} Q_{v-\frac{1}{2}}^{(1)}(\cos \theta)+i e^{-i \pi v} Q_{\nu-\frac{1}{2}}^{(2)}(\cos \theta)
$$

where asymptotically

$$
Q_{v-\frac{1}{2}}^{(1,2)}(\cos \theta) \sim \frac{e^{\mp i(v \theta-\pi / 4)}}{(2 \pi v \sin \theta)^{\frac{1}{2}}}
$$

for $\varepsilon \leqq \theta \leqq \pi-\varepsilon,|v| \gg 1$, and $|v| \varepsilon \gg 1$ (Nussenzveig, 1965). Substituting (36) together with the expansion

$$
[\cos (v \pi)]^{-1}=2 e^{ \pm i v \pi} \sum_{n=0}^{\infty}(-1)^{n} e^{ \pm 2 i \pi v \eta}
$$

into the integral (30), we obtain

$$
\begin{aligned}
\psi_{>}(r, \theta)= & \sum_{n=0}^{\infty}\left[\int_{c_{2}}(-1)^{n} \hat{\psi}_{>}(v) Q_{\nu-\frac{1}{2}}^{(2)}(\cos \theta) e^{i 2 \pi v \eta} \gamma d v\right. \\
& \left.-\int_{c_{1}}(-1)^{n} \hat{\psi}_{>}(v) Q_{v-\frac{1}{2}}^{(1)}(\cos \theta) e^{-i 2 \pi v \eta} v d v\right] .
\end{aligned}
$$

From the asymptotic representation of $Q_{v-\frac{1}{2}}^{(1,2)}$ it is evident that the $n$th term in the first integral represents waves that have traveled around the Earth $n$ times in the $+\theta$ direction. Thus, direct body waves propagating in the $+\theta$ direction are represented by the first integral with $n=0$, or

$$
\psi_{>}(r, \theta)=\int_{c_{2}} \hat{\psi}_{>} Q_{v-\frac{1}{2}}^{(2)}(\cos \theta) v d v
$$

We next introduce the Debye asymptotic forms for spherical Hankel functions as given by Nussenzeig (1965)

where

$$
\begin{aligned}
& h_{l}^{(1)}(\zeta) \sim \zeta^{-\frac{1}{2}}\left(\zeta^{2}-v^{2}\right)^{-\frac{1}{4}} e^{i \chi(\zeta)} \\
& h_{l}^{(2)}(\zeta) \sim \zeta^{-\frac{1}{2}}\left(\zeta^{2}-v^{2}\right)^{-\frac{1}{4}} e^{-i \chi(\zeta)}
\end{aligned}
$$

$$
\begin{aligned}
x(\zeta) & =\left(\zeta^{2}-v^{2}\right)^{\frac{1}{2}}-v \cos ^{-1}(v / \zeta)-\pi / 4 \\
v & =l+\frac{1}{2}, \quad v<\zeta .
\end{aligned}
$$

The corresponding asymptotic forms of the derivatives are

$$
\begin{aligned}
& h_{l}{ }^{(1)^{\prime}}(\zeta) \sim i\left(1-v^{2} / \zeta^{2}\right)^{\frac{1}{2}} h_{l}{ }^{(1)}(\zeta) \\
& h_{l}^{(2)^{\prime}}(\zeta) \sim-i\left(1-v^{2} / \zeta^{2}\right)^{\frac{1}{2}} h_{l}{ }^{(2)}(\zeta) .
\end{aligned}
$$

Accordingly the terms $h_{1}$ and $h_{2}$ in (34) become

$$
\begin{aligned}
& h_{1} \sim-\frac{i}{k_{0}}\left(k_{0}^{2}-\kappa^{2}\right)^{\frac{1}{2}}=-\frac{i}{k_{0}} \eta \\
& h_{2} \sim \frac{i}{k_{0}}\left(k_{0}^{2}-\kappa^{2}\right)^{\frac{1}{2}}=\frac{i}{k_{0}} \eta
\end{aligned}
$$


and $V$ becomes

$$
V \sim \frac{\Phi(v)\left[i m \eta+\frac{\rho_{1}^{\prime}}{2 \rho_{1}}-\frac{1}{2 a}\right]+\Phi^{\prime}(v)}{\Phi(v)\left[i m \eta-\frac{\rho_{1}^{\prime}}{2 \rho_{1}}+\frac{1}{2 a}\right]-\Phi^{\prime}(v)}
$$

where the primes here indicate differentiation with respect to $z, m=\rho_{1} / \rho_{0}$ and $\eta=$ $\left(k_{0}{ }^{2}-\kappa^{2}\right)^{\frac{1}{2}}$. This is equivalent to the reflection coefficient for plane waves incident on a plane boundary when the transformed spherical boundary conditions are used and $\Phi(v)$ is a solution to (20) in the lower medium. If the natural boundary conditions for a plane boundary are used, the term $(1 / 2 \mathrm{a})$ will be absent, and $V$ will be the true plane wave-plane boundary reflection coefficient. As indicated above, for crustal and upper-mantle body waves, $|\kappa a| \gg 1$ and the true plane-wave reflection coefficient can be used in (38) without significant loss of accuracy.

Introducing the earth-flattening transformation (21) into the asymptotic forms of the spherical Hankel functions (39) and substituting this in the expression for $\hat{\psi}_{>}(v)(35)$, we obtain

$$
\hat{\psi}_{>}(\kappa) \sim \frac{1}{a^{2}\left(1+\delta_{1}\right)}\left\{\frac{i}{\eta} e^{-i \eta\left(z-z_{0}\right)\left(1+\delta_{2}\right)}+V \frac{i}{\eta} e^{i \eta\left(z+z_{0}\right)\left(1+\delta_{3}\right)}\right\}
$$

where the $\delta_{i}$ are terms of $O(z / a)$ given by

$$
\begin{aligned}
& \delta_{1}=\frac{\kappa^{2}+2 \eta^{2}}{\eta^{2}} \frac{\left(z+z_{0}\right)}{a}+O\left(z^{2} / a^{2}\right) \\
& \delta_{2}=\frac{\kappa^{2}}{\eta^{2}} \frac{\left(z+z_{0}\right)}{a}+O\left(z^{2} / a^{2}\right) \\
& \delta_{3}=\frac{\kappa^{2}}{\eta^{2} a}\left(\frac{z_{0}{ }^{2}+z^{2}}{z_{0}+z}\right)+O\left(z^{2} / a^{2}\right) .
\end{aligned}
$$

Note that if the $\delta_{i}$ are are neglected, the first and second terms in the brackets reduce to the expression for the direct and reflected fields, respectively, in the case of a point source over a plane boundary. If the terms of $O\left(z^{2} / a^{2}\right)$ are neglected, the $\delta_{i}$ become first order corrections for boundary curvature corresponding to the linear approximation to the earth-flattening transformation (23).

We are primarily interested in the integral representation of the reflected field, which by (38) and (42) can be written as

$$
\psi_{>r} \sim i \int_{c_{2^{1}}} Q_{\kappa a-\frac{1}{2}}^{(2)}(\cos \theta) V(\kappa) \frac{e^{i \eta\left(z+z_{0}\right)\left(1+\delta_{2}\right)}}{\eta\left(1+\delta_{1}\right)} \kappa d \kappa
$$

for $|z / a|<1$. Here the variable of integration has been changed to $\kappa$ according to $v=\kappa a$ and the path $c_{2}$ lies just above the real $\kappa$ axis and extends from $-\infty$ to $+\infty$. The integral is now in a form closely analogous to the Weyl integral (31). In fact, as the curvature of the boundary $(1 / a)$ goes to zero, the integral reduces to the Weyl integral. To see that this is so, we note that

$$
Q_{v}{ }^{(2)}(\cos \theta)=\frac{1}{2}\left[P_{v}(\cos \theta)-\frac{2 i}{\pi} Q_{v}(\cos \theta)\right]
$$


(Nussenzveig, 1965). Furthermore, for $\theta>0$

$$
\begin{aligned}
& \operatorname{Lim}_{v \rightarrow \infty}\left\{P_{v}(\cos \theta)\right\}=J_{0}(v \theta) \\
& \left.\operatorname{Lim}_{v \rightarrow \infty}\left\{Q_{\nu} \cos \theta\right)\right\}=-\frac{\pi}{2} Y_{0}(v \theta)
\end{aligned}
$$

(Abramowitz and Stegun, 1964). From this

and

$$
\operatorname{Lim}_{v \rightarrow \infty}\left\{Q_{v}{ }^{(2)}(\cos \theta)\right\}=\frac{1}{2}\left[J_{0}(v \theta)+i Y_{0}(v \theta)\right]=\frac{1}{2} H_{0}{ }^{(1)}(v \theta)
$$

where

$$
\operatorname{Lim}_{v \rightarrow \infty}\left\{\psi_{>r}\right\}=\frac{i}{2} \int_{-\infty}^{\infty} H_{0}^{(1)}(v \theta) V_{p}(\kappa) e^{i \eta\left(z+z_{0}\right)} \frac{\kappa}{\eta} d \kappa
$$

$$
\nu \theta=\kappa a \theta \rightarrow \kappa \rho .
$$

In general, neither Weyl integral (31) nor (43) can be evaluated exactly. The usual practice in wave-propagation problems is to consider the far-field solution using the large-argument asymptotic form of the Hankel function (see Brekhovskikh, 1960). This puts the Weyl integral in a form that is amenable to evaluation by approximate techniques such as the method of steepest descents. In particular, we have for $|\kappa \rho| \gg 1$

$$
H_{0}^{(1)}(\kappa \rho) \sim\left(\frac{2}{\pi \kappa \rho}\right)^{\frac{1}{2}} e^{i(\kappa \rho-\pi / 4)}
$$

so that the Weyl integral becomes

$$
\psi \sim e^{i \pi / 4} \sqrt{\frac{1}{2 \pi \rho}} \int_{-\infty}^{\infty} V_{p}(\kappa) e^{i\left[\kappa \rho+\eta\left(z+z_{0}\right)\right]} \frac{\kappa^{\frac{1}{2}}}{\eta} d \kappa
$$

Using the large-order asymptotic form for the Legendre function (37) in the analogous integral for a spherical boundary (43), we have

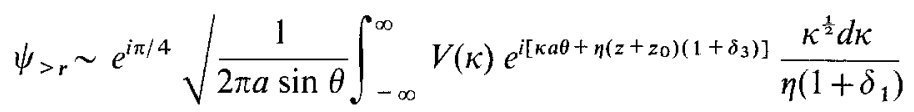

for $\varepsilon \leqq \theta \leqq \pi-\varepsilon,|\kappa a| \gg 1$ and $|\kappa a| \varepsilon \gg 1$.

Equation (45) is the far-field integral expression for waves generated at a steady point source and reffected by a radially heterogeneous medium in $r<a$. It gives a valid description of the reflected wave field under the conditions: (a) $|z / a| \ll 1$, the source and receiver distances above the boundary at $r=a$ are small with respect to $a$, (b) $|\kappa a| \gg 1$ and $|\eta a| \gg 1$, the wavelength is much less than the radius, $a$, and both near-normal and near-grazing angles of incidence are avoided; and (c) $\theta \geqq \varepsilon$ and $|\kappa a| \varepsilon \gg 1$; solutions are valid at angular distances greater than several wavelengths from the source (the far-field approximation).

\section{Discussion}

We have developed an exact earth-flattening transformation for the class of wave problems that can be formulated in terms of uncoupled, scalar Helmholtz equations in spherically symmetric, radially heterogeneous media. This class includes acoustic and torsional $(\mathrm{SH})$ elastic motion provided only that density or shear modulus gradients are small with respect to wavelengths considered. It also includes high-frequency elastic waves with either $P$ or $S V$ motion, although general spherical (or coupled $P-S V$ ) elastic motion is excluded. 
The earth-flattening transformation involves the mapping of spherical curvature into an exponential velocity profile according to (22) and an exponential relation between the radial coordinate, $r$, and the vertical coordinate, $z$, according to (21). In the absence of sources, this transformation is analogous to those developed for elastic $S H$ motion by Anderson and Toksöz (1963), Satô (1968), and Biswas and Knopoff (1970). As such, the transformation can be used to study torsional normal modes or Love waves in a radially heterogeneous earth in terms of plane, vertically heterogeneous layered models. For this application, the Thomson-Haskell matrix formalism together with the properly transformed spherical boundary conditions can be used to advantage.

In the presence of a point source, the wavefield scattered by a spherically symmetric, radially heterogeneous body is expressed as an infinite sum of eigenfunctions over discrete wave numbers. By operating on the radial and angular parts of the solution with the earth-flattening transformation and the Watson transform, respectively, the solution is converted to an integral over continuous wave numbers in an equivalent flat, vertically heterogeneous model. The far-field form of this integral representation has many properties in common with the far-field form of the Weyl integral. As such, it offers a convenient means for the evaluation of body wave-propagation problems in the outer layers of a spherical earth. (The exact integral representation in flat coordinates offers no advantage for either numerical or analytical evaluation over the usual equivalent representation in spherical coordinates obtained by applying the Watson transform alone.)

Comparing (45) with (44), we can see how curvature modifies the flat representation of the reflected field. In particular: (a) The horizontal distance, $\rho$, is replaced by $a \sin \theta$ and $a \theta$ (arc distance) in the geometric factor in front of the integral and in the exponential term inside the integral, respectively. Clearly for small angular distances $a \sin \theta \simeq a \theta \simeq \rho$. (b) The factors $\left(1+\delta_{i}\right)$ multiplying the terms containing the vertical wave number, $\eta$, correspond to corrections to the angle of incidence at the boundary $r=a$ due to curvature of the boundary. For moderate angles of incidence and for $\left|z_{0} / a\right| \ll 1$ these factors will be nearly unity and can be neglected. Note that by omitting the $\delta_{i}$, we are only neglecting the effects of curvature above the boundary $r=a$; the effects of curvature below the boundary are contained in the reflection coefficients, $V(\kappa)$. (c) The reflection coefficient $V(\kappa)$ given by (41) is the plane-wave reflection coefficient for the half-space $z<0(r<a)$ in which the velocity profile is modified by the exponential factor $e^{-z / a}$ according to (22) and the transformed spherical boundary conditions are used. Because the function $\Phi(v)$, occurring in the reflection coefficient, is the exact radial eigenfunction for $r<a$ (expressed in the vertical coordinate $z$ ), the integral (45) can in principle be used to obtain the wave field reflected from arbitrary depths below the boundary $r=a$.

In general, the analytic evaluation of (45) for realistic earth models requires that additional approximations be made. Closed form expressions for the radial eigenfunction, $\Phi$, can be obtained for very few velocity distributions, and normally it is necessary to approximate the reflection coefficient using one of the several methods described in Brekhovskikh (1960). For example, it seems that this integral representation (45), when coupled with the Epstein method for evaluating flat problems with continuous velocity variations (Epstein, 1930; also see Phinney, 1970 for a discussion of geophysical applications of the Epstein method), provides a promising approach for studying waves reflected from a variety of interesting transition zones in a spherical earth. An example of a specific application of (45) is given by Hill (1971). Here, the integral is used to determine the effects of curvature and velocity gradients on critically refracted waves in the crust and upper mantle. In this case, the effects of velocity gradients and curvature just beneath the refracting boundary dominate, and it is possible to use the linear approximation to the earth-flattening transformation (23). 


\section{ACKNOWLEDGMENTS}

I would like to thank C. B. Archambeau for his advice and encouragement and D. L. Anderson and P. G. Richards for many helpful discussions during this study. Some of the results presented in this paper are contained in a thesis submitted in partial satisfaction of requirements for a Ph.D. degree at the California Institute of Technology. This research was supported in part by the Air Force Office of Scientific, Research under contract AFOSR F44620-69-C-0067.

\section{REFERENCES}

Abramowitz, M., and I. A. Stegun (1964). Handbook of Mathematical Functions, U.S. Nat'1 Bur. Standards.

Alterman, Z., H. Jarosch, and G. L. Pekeris (1961), Propagation of Rayleigh waves in the earth, Geophys. J. 4, 219-241.

Anderson, D. L. and M. N. Toksöz (1963). Surface waves on a spherical earth, J. Geophys. Res. 68, 3483-3500.

Biswas, N. N. and L. Knopoff (1970). Exact earth-flattening calculations for Love waves, Bull. Seism, Soc. Am. 60, 1123-1137.

Brekhovskikh, L. M. (1960), Waves in Layered Media, Academic Press, New York, 561 pp.

Bremmer, H. (1949). Terrestrial Radio Waves, American Elsevier Publ. Co., New York, 343 pp.

Budden, K. G. (1960). The Wave-Guide Mode Theory of Wave Propagation, Prentice-Hall, Englewood, N.J.

Chapman, C. H. (1969). Seismic wave diffraction theory, Ph.D. Thesis, Christ's College, Cambridge, 212 p.

Epstein, P. S. (1930). Reflection of waves in an inhomogeneous absorbing medium, Proc. Natl. Acad. Sci. Wash. 16, p. 627.

Gilbert, F. and D. V. Helmberger (1971). Generalized ray theory for a layered sphere, Geophys. J. (in press).

Grant, F. S. and G. F. West (1965). Interpretation Theory in Applied Geophysics, McGraw-Hill, New York, $584 \mathrm{p}$.

Hill, D. P. (1971). High frequency wave propagation in the Earth: theory and observation, Ph.D. Thesis, California Institute of Technology, $390 \mathrm{p}$.

Kovach, R. L. and D. L. Anderson (1962). Long-period Loves waves in a heterogeneous spherical earth, J. Geophys. Res. 67, 5243-5255.

Muller, G. (1971). Approximate treatment of elastic body waves in media with spherical symmetry, Geophys. J. 23, 435-449.

Nussenzveig, H. M. (1965). High frequency scattering by an impenetrable sphere, Ann. Phys. (Paris) 34 23-95.

Phinney, R. A. (1970). Reflection of acoustic waves from a continuously varying interfacial region, Rev. Geophys. Space. Phys. 8, 517-532.

Phinney, R. A. and S. S. Alexander, (1966), $P$ wave diffraction theory and the structure of the coremantle boundary, J. Geophys. Res. 71, 5959-5975.

Richards, P. G. (1971). Potentials for elastic displacement in spherically symmetric media, J. Acoust. Soc. Am. 50, 188-197.

Satô, R. (1968b), Effect of spherical crustal layering ( $S H$ waves), J. Phys. Earth 16, 1-6.

Schelling, S. G., G. R. Burrows, and F. B. Ferrell (1933). Ultrashort period wave propagation, Proc. I.R.E. 21, 427-463.

Sholte, J. G. J. (1956). On seismic waves in a spherical earth, Komnkl. Ned. Meterol. Inst. Pub. 65, North-Holland.

U.S. Geological Survey

Menlo Park, California 94025

and

Division of Geological and Planetary Sciences

CALIFornia Institute of TECHNOLOGY

Pasadena, California 91109

CONTRiBution No. 2137

Manuscript received February 24, 1972 